An International Journal of Optimization and Control: Theories \& Applications ISSN: 2146-0957 eISSN: 2146-5703

Vol.7, No.3, pp.281-287 (2017)

https://doi.org/10.11121/ijocta.01.2017.00492

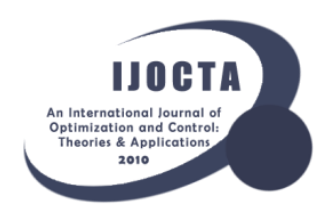

\title{
Numerical approach for solving time fractional diffusion equation
}

\author{
Dilara Altan Koç, Mustafa Gülsu \\ Department Mathematics, Mugla Sitki Kocman University, Turkey \\ dilaraaltan@mu.edu.tr,mgulsu@mu.edu.tr
}

\section{ARTICLE INFO}

Article history:

Received:16 June 2017

Accepted: 13 November 2017

Available Online: 21 November 2017

Keywords:

Diffusion equation

Finite difference schemes

Explicit method

\begin{abstract}
In this article one of the fractional partial differential equations was solved by finite difference scheme based on five point and three point central space method with discretization in time. We use between the Caputo and the Riemann-Liouville derivative definition and the Grünwald-Letnikov operator for the fractional calculus. The stability analysis of this scheme is examined by using von-Neumann method. A comparison between exact solutions and numerical solutions is made. Some figures and tables are included.
\end{abstract}

AMS Classification 2010:

65N06, 65M06

(c) $)$ EY

\section{Introduction}

Anomalous equation is a diffusion process with a nonlinear relationship to time contrary to typical diffusion process, connected with the interactions within the complex and non- homogeneous background. In contrast to typical diffusion, anomalous diffusion is described by a power law [1]. Anomalous diffusion has an important role in the literature to describe many physical phenomena, crowded systems or diffusion through porous media. On the other hand this phenomena is observed in heat baths [2], diffusion through also porous material $[3,4]$, nuclear magnetic resonance diffusometry in disordered materials [4], behaviour of polymers in a glass transition [5,6]and also particle dynamics inside polymer network [7].Fractional order linear and nonlinear differential equations were examined by some researchers by using different methods [8-12]. In this study we consider the fractional subdiffusion equation in the following form [13]:

${ }_{0}^{c} D_{t}^{\alpha} U(x, t)=K_{\alpha} \frac{\partial^{2}}{\partial x^{2}} U(x, t)+f(x, t)$,

$t \in[0, T], x \in[0, L]$,

where $0<\alpha<1, U(x, t)$ is a field variable (probability density of diffusive displacements $x$ in a time t), $K_{\alpha}$ is the diffusion coefficient, ${ }_{0}^{c} D_{t}^{\alpha}$ is the fractional derivative in the Caputo sense, $\alpha$ is a real order of this operator and $f(x, t)$ is a suitable right hand side function. The time fractional subdiffusion equation has been obtained by replacing the time derivative in ordinary diffusion by a fractional order. In a typical diffusion process $\alpha=1$, if $0<\alpha<1$ the particle undergoes subdiffusion, if $1<\alpha<2$ the phenomena is called super diffusion equation. Recently, because of their practical applications, anomalous subdiffusion equation has received much attention. The common methods for solving fractional-order equations are purely mathematical [14], in terms of Mittag Lefler function [15], Green's function solution [16], numerical methods [17], variational iteration method [18] and differential transformations [19]. In the present work we concentrate on one of the numerical methods which is fractional finite difference method for solutions of equation (1).

In section 2, we give some information about fractional calculus, some definition of fractional derivative which are used in the next sections. We talk about finite difference method, stability of this explicit scheme and calculate the maximum absolute error ( $\left.E_{\max }\right)$ at the time $t$ and the root mean square error $E_{R M S}$ at the same time in section 3. In the last section 4, we apply the method to fractional diffusion equation with the given initial and boundary conditions and compared the numerical solutions with the exact results.

${ }^{*}$ Corresponding author 


\section{Basic concepts of fractional calculus}

In this paper we consider the fractional partial differential Eq.(1), with the boundary and initial-value conditions:

$U(0, t)=g_{0}(t), \quad U(L, t)=g_{L}(t)$,

$U(x, 0)=p_{0}(x)$.

Fractional calculus includes different definition of fractional derivative operator such as RiemannLiouville derivative, the Caputo derivative, the Grünvald Letnikov derivative, the Riesz derivative and also the Weyl-Marchaud derivative.

We introduced the definition of Caputo operator [1], ${ }_{0}^{c} D_{t}^{\alpha} U(x, t)=\frac{{ }^{c} \partial^{\alpha}}{\partial t^{\alpha}} U(x, t) \square \frac{1}{\Gamma(m+1-\alpha)} \int_{0}^{t} \frac{\frac{\partial^{m+1}}{\partial \xi^{m+1}} U(x, \xi)}{(t-\xi)^{\alpha-m}} d \xi$,

, $\alpha \notin \mathrm{N} m=[\alpha],[$.$] denotes an integer part of a real$ number. The operator ${ }_{0} D_{t}^{\alpha}$ is defined in the Riemann-Liouville sense as [1],

$$
{ }_{0} D_{t}^{\alpha} U(x, t)=\frac{\partial^{\alpha}}{\partial t^{\alpha}} U(x, t) \square \frac{1}{\Gamma(m+1-\alpha)} \frac{\partial^{m+1}}{\partial t^{m+1}} \int_{0}^{t} \frac{U(x, \xi)}{(t-\xi)^{\alpha-m}} d \xi .
$$

According to fractional calculus between the Caputo and the Riemann-Liouville derivative is following form [1],

$$
{ }_{0}^{c} D_{t}^{\alpha} U(x, t)={ }_{0} D_{t}^{\alpha} \mathrm{U}(\mathrm{x}, \mathrm{t})-\sum_{\mathrm{k}=0}^{\mathrm{m}} \frac{\mathrm{t}^{\mathrm{k}-\alpha}}{\Gamma(\mathrm{k}-\alpha+1)} \frac{\partial^{k}}{\partial t^{k}} U(x, 0) .
$$

We divide the spatial domain $[0, L]$ into the uniform mesh with $N$ subintervals. $x_{i}=i \Delta x,(\Delta x=h)$, for $i=0, \ldots, N$ where, $h=\frac{L}{N}$. The time interval $[0, T]$ is divided into $M$ subintervals, where the subinterval length equals to $k=\frac{T}{M}, t_{j}=j \Delta t,(\Delta t=k)$, for $j=0, \ldots, M$. We mention another definition of the fractional derivative which is Grünwald-Letnikov sense as the following [1]:

${ }_{0}^{G L} D_{t}^{\alpha} U(x, t)=\lim _{\Delta t \rightarrow 0}(\Delta t)^{-\alpha} \sum_{j=0}^{[t / \Delta t]}(-1)^{j}\left(\begin{array}{c}\alpha \\ j\end{array}\right) U(x, t-j \Delta t)$.

This definition is equivalent to the Riemann-Liouville definition (3). However the Grünwald-Letnikov operator is more suitable and more obvious in numerical calculations.

The Grünwald-Letnikov operator Eq.(6) is approximated with the interval $[0, \mathrm{~T}]$ with the subinterval length $\Delta t$ as [1],

$$
{ }_{0}^{G L} D_{t}^{\alpha} U(x, t) \approx \sum_{j=0}^{[t / \Delta t]} c_{j}^{(\alpha)} U(x, t-j \Delta t),(7)
$$

where $c_{j}^{(\alpha)}=(\Delta t)^{-\alpha}(-1)^{j}\left(\begin{array}{c}\alpha \\ j\end{array}\right)$ for $j=0,1, \ldots$.

By using the recurrence relationship we obtain,

$$
c_{0}^{(\alpha)}=(\Delta t)^{-\alpha}, c_{1}^{(\alpha)}=-\alpha(\Delta t)^{-\alpha},
$$

$$
c_{j}^{(\alpha)}=\left(1-\frac{1+\alpha}{j}\right) c_{j-1}^{(\alpha)} j=1,2, \ldots .
$$

These coefficients come from the generating function [22],

$$
(1-z)^{\alpha}=\sum_{k=0}^{\infty} c_{k}^{(\alpha)} z^{k}
$$

If we put Eq.(7) in place of ${ }_{0} D_{t}^{\alpha} U(x, t)$ operator in Eq.(5), we obtain the following equation, ${ }_{0} D_{t}^{\alpha} \mathrm{u}\left(\mathrm{x}_{\mathrm{i}}, \mathrm{t}_{\mathrm{j}}\right)=\sum_{w=0}^{j} c_{w}^{(\alpha)} u\left(x_{i}, t_{j-w}\right)-\sum_{q=0}^{m} \frac{\mathrm{t}_{\mathrm{j}}{ }^{\mathrm{q}-\alpha}}{\Gamma(\mathrm{q}-\alpha+1)} p_{q}\left(x_{i}\right)$. (10)

We will use this fractional derivative definition for the left-side of Eq (1).

\section{Finite difference scheme of the fractional diffusion equation}

In this work we take initial value conditions as in the Eq.(2). If we consider them as discrete forms $x=x_{i}$, $i=1, \ldots, N-1 \quad$ and $t=t_{j}, j=0, \ldots, m$ we obtain $u\left(x_{i}, t_{0}\right)=p_{0}(i h)$. The second derivative at the space in the right side of Eq.(1) in terms of 5-point central differences can be written as,

$$
\begin{aligned}
& \frac{\partial^{2}}{\partial x^{2}} u\left(x_{i}, t\right)=\frac{1}{12 h^{2}}\left(-u\left(x_{i-2}, t\right)+16 u\left(x_{i-1}, t\right)\right. \\
& \left.-30 u\left(x_{i}, t\right)+16 u\left(x_{i+1}, t\right)-u\left(x_{i+2}, t\right)\right),
\end{aligned}
$$

for $i=2, \ldots, 8$.

In case of $i=1$ and $i=9$ for the second derivative at the space we can use the following 3-point central difference formula,

$\frac{\partial^{2}}{\partial x^{2}} u\left(x_{i}, t\right)=\frac{u\left(x_{i+1}, t\right)-2 u\left(x_{i}, t\right)+u\left(x_{i-1}, t\right)}{h^{2}}$.

Assuming $t=t_{j-1}$ and substituting Eq.(10) and Eq.(11) into Eq. (1) we obtain,

$$
\begin{aligned}
& \sum_{w=0}^{j} c_{w}^{(\alpha)} u\left(x_{i}, t_{j-w}\right)-\sum_{q=0}^{m} \frac{\mathrm{t}_{\mathrm{j}}{ }^{\mathrm{q}-\alpha}}{\Gamma(\mathrm{q}-\alpha+1)} p_{q}\left(x_{i}\right) \\
& =\frac{1}{12 h^{2}}\left(-u\left(x_{i-2}, t_{j-1}\right)\right. \\
& +16 u\left(x_{i-1}, t_{j-1}\right)-30 u\left(x_{i}, t_{j-1}\right) \\
& \left.+16 u\left(x_{i+1}, t_{j-1}\right)-u\left(x_{i+2}, t_{j-1}\right)\right)+f\left(x_{i}, t_{j}\right) .
\end{aligned}
$$

Disintegrating partially into terms the first sum in Eq.(13) we have,

$$
\begin{aligned}
& c_{0}^{(\alpha)} u\left(x_{i}, t_{j}\right)+c_{1}^{(\alpha)} u\left(x_{i}, t_{j-1}\right)+\sum_{w=2}^{j} c_{w}^{(\alpha)} u\left(x_{i}, t_{j-w}\right) \\
& -\sum_{q=0}^{m} \frac{\mathrm{t}_{\mathrm{j}}^{\mathrm{q}-\alpha}}{\Gamma(\mathrm{q}-\alpha+1)} p_{q}\left(x_{i}\right)=\frac{1}{12 h^{2}}\left(-u\left(x_{i-2}, t_{j-1}\right)\right. \\
& +16 u\left(x_{i-1}, t_{j-1}\right)-30 u\left(x_{i}, t_{j-1}\right)+16 u\left(x_{i+1}, t_{j-1}\right) \\
& \left.-u\left(x_{i+2}, t_{j-1}\right)\right)+f\left(x_{i}, t_{j}\right) .
\end{aligned}
$$


If we write more clearly,

$$
\begin{aligned}
& (\Delta t)^{-\alpha}(-1)^{0}\left(\begin{array}{c}
\alpha \\
0
\end{array}\right) u\left(x_{i}, t_{j}\right)+(\Delta t)^{-\alpha}(-1)^{1}\left(\begin{array}{c}
\alpha \\
1
\end{array}\right) u\left(x_{i}, t_{j-1}\right) \\
& +\sum_{w=2}^{j} c_{w}^{(\alpha)} u\left(x_{i}, t_{j-w}\right)-\sum_{q=0}^{m} \frac{\mathrm{t}_{\mathrm{j}}^{(\mathrm{q}-\alpha)}}{\Gamma(\mathrm{q}-\alpha+1)} p_{q}\left(x_{i}\right) \\
& =\frac{1}{12 h^{2}}\left(-u\left(x_{i-2}, t_{j-1}\right)\right. \\
& +16 u\left(x_{i-1}, t_{j-1}\right)-30 u\left(x_{i}, t_{j-1}\right)+16 u\left(x_{i+1}, t_{j-1}\right) \\
& \left.-u\left(x_{i+2}, t_{j-1}\right)\right)+f\left(x_{i}, t_{j}\right) .
\end{aligned}
$$

Denoting

$$
u\left(x_{i}, t_{j}\right)=u_{i, j}, p_{q}\left(x_{i}\right)=p_{q, i} \text { and }
$$

$f\left(x_{i}, t_{j}\right)=f_{i, j}$ and we have,

$u_{i, j}=(\Delta t)^{\alpha}\left(\left(\frac{\alpha}{(\Delta t)^{\alpha}}-\frac{30}{12 h^{2}}\right) u_{i, j-1}-\sum_{w=2}^{j} c_{w}^{(\alpha)} u_{i, j-w}\right.$

$+\sum_{q=0}^{m} \frac{\mathrm{t}_{\mathrm{j}}^{(\mathrm{q}-\alpha)}}{\Gamma(\mathrm{q}-\alpha+1)} p_{q, i}$

$+\frac{1}{12 h^{2}}\left(-u_{i-2, j-1}+16 u_{i-1, j-1}\right.$

$\left.\left.+16 u_{i+1, j-1}-u_{i+2, j-1}\right)+f_{i, j}\right)$,

for $i=2, \ldots, N-2, j=1, \ldots, M$.

To obtain the formula of $u_{i, j}$ for $i=1$ and $i=9$, we have to use 3-point central differences. Substituting Eq.(10) and Eq.(12) into Eq.(1) we obtain,

$$
\begin{aligned}
& \sum_{w=0}^{j} c_{w}^{(\alpha)} u\left(x_{i}, t_{j-w}\right)-\sum_{q=0}^{m} \frac{\mathrm{t}_{\mathrm{j}}{ }^{\mathrm{q}-\alpha}}{\Gamma(\mathrm{q}-\alpha+1)} p_{q}\left(x_{i}\right) \\
& =\frac{u\left(x_{i+1}, t\right)-2 u\left(x_{i}, t\right)+u\left(x_{i-1}, t\right)}{h^{2}}+f\left(x_{i}, t_{j}\right) .
\end{aligned}
$$

Disintegrating partially into terms the first sum in Eq.(17) and forming we obtain the following equation;

$$
\begin{aligned}
& c_{0}^{(\alpha)} u\left(x_{i}, t_{j}\right)+c_{1}^{(\alpha)} u\left(x_{i}, t_{j-1}\right)+\sum_{w=2}^{j} c_{w}^{(\alpha)} u\left(x_{i}, t_{j-w}\right) \\
& -\sum_{q=0}^{m} \frac{\mathrm{t}_{\mathrm{j}}^{\mathrm{q}-\alpha}}{\Gamma(\mathrm{q}-\alpha+1)} p_{q}\left(x_{i}\right) \\
& =\frac{u\left(x_{i+1}, t\right)-2 u\left(x_{i}, t\right)+u\left(x_{i-1}, t\right)}{h^{2}}+f\left(x_{i}, t_{j}\right),
\end{aligned}
$$

and we have,

$$
\begin{aligned}
& (\Delta t)^{-\alpha}(-1)^{0}\left(\begin{array}{l}
\alpha \\
0
\end{array}\right) u\left(x_{i}, t_{j}\right)+ \\
& (\Delta t)^{-\alpha}(-1)^{1}\left(\begin{array}{c}
\alpha \\
1
\end{array}\right) u\left(x_{i}, t_{j-1}\right) \\
& +\sum_{w=2}^{j} c_{w}^{(\alpha)} u\left(x_{i}, t_{j-w}\right)-\sum_{q=0}^{m} \frac{\mathrm{t}_{\mathrm{j}}^{(\mathrm{q}-\alpha)}}{\Gamma(\mathrm{q}-\alpha+1)} p_{q}\left(x_{i}\right) \\
& =\frac{u\left(x_{i+1}, t\right)-2 u\left(x_{i}, t\right)+u\left(x_{i-1}, t\right)}{h^{2}}+f\left(x_{i}, t_{j}\right),
\end{aligned}
$$

Denoting $u\left(x_{i}, t_{j}\right)=u_{i, j} \quad, \quad p_{q}\left(x_{i}\right)=p_{q, i} \quad$ and $f\left(x_{i}, t_{j}\right)=f_{i, j}$,we have,

$u_{i, j}=(\Delta t)^{\alpha}\left[\left(\frac{\alpha}{(\Delta t)^{\alpha}}-\frac{2}{h^{2}}\right) u_{i, j-1}-\sum_{w=2}^{j} c_{w}^{(\alpha)} u_{i, j-w}\right.$

$\left.+\sum_{q=0}^{m} \frac{\mathrm{t}_{\mathrm{j}}^{(\mathrm{q}-\alpha)}}{\Gamma(\mathrm{q}-\alpha+1)} p_{q, i}+\frac{1}{h^{2}}\left(u_{i+1, j-1}+u_{i-1, j-1}\right)+f_{i, j}\right]$, for $i=1$ and $i=9, j=1, \ldots, M$.

As usual for explicit methods, the present explicit difference schemes Eq.(16) and Eq.(20) is not unconditionally stable. Therefore it is important to determine the conditions under which these schemes are stable.

\subsection{Stability of method}

The present explicit difference schemes, Eq.(16) and Eq.(20) is not unconditionally stable. Therefore it is important to determine the conditions under which the method is stable. We will employ that fractional von Neumann stability (or Fourier stability) analysis put forward in [22]. We start by assuming a solution of our problem with the form $u_{i}^{(j)}=\varsigma^{q} e^{i \beta p h}$, where $q$ is a real spatial number. Inserting this expression into Eq.(16) we get,

$\varsigma^{q}=(\Delta t)^{\alpha}\left[\left(\frac{\alpha}{(\Delta t)^{\alpha}}-\frac{30}{12 h^{2}}\right) \varsigma^{q-1}-\sum_{\omega=2}^{j} c_{\omega}^{(\alpha)} \varsigma^{q-\omega}\right.$

$+\sum_{q=0}^{m} \frac{\mathrm{t}_{\mathrm{j}}^{(\mathrm{q}-\alpha)}}{\Gamma(\mathrm{q}-\alpha+1)} p_{q, i}$

$\left.+\frac{1}{12 h^{2}} \varsigma^{q-1}\left(-e^{-2 i \beta h}+16 e^{-i \beta h}+16 e^{i \beta h}-e^{2 i \beta h}\right)+f_{i, j}\right]$.

In this study, because of the order of fractional differential equation $m=0$ then, $p_{0}^{j}$ and the source term is neglected $(f(x, t)=0)$ and the stability of the process is determined by the behaviour of $\varsigma^{q}$. Writing

$\varsigma^{q+1}=\xi \varsigma^{q},(22)$

and assuming $\xi$ is independent of time, we get, 
$1=(\Delta t)^{\alpha}\left(\frac{\alpha}{(\Delta t)^{\alpha}}-\frac{30}{12 h^{2}}\right) \xi^{-1}-(\Delta t)^{\alpha} \sum_{\omega=2}^{j} c_{\omega}^{(\alpha)} \xi^{-\omega}$

$+S \xi^{-1}\left(30+4 \sin ^{2}(\beta h)-32 \sin ^{2}\left(\frac{\beta h}{2}\right)\right)$,

where $S=\frac{(\Delta t)^{\alpha}}{12 h^{2}}$.

If $|\xi|>1$ for some $q$, the temporal factor of the solution grows to infinity according to Eq.(22) and the mode is unstable. Considering the extreme value $\xi=-1$, we obtain from Eq.(23) the following stability bound on $S$ [22]: $S\left(30+4 \sin ^{2}(\beta h)-32 \sin ^{2}\left(\frac{\beta h}{2}\right)\right)$

$\leq(\Delta t)^{\alpha}\left(\frac{30}{12 h^{2}}-\frac{\alpha}{(\Delta t)^{\alpha}}-\sum_{\omega=2}^{j} c_{\omega}^{(\alpha)}(-1)^{\omega}\right)-1 \equiv S_{j}^{x}$.

$S_{j}^{x}$ approaches $S^{x} \equiv \lim _{j \rightarrow \infty} S_{j}^{x}$. The value of $S^{x}$ can be concluded from Eq.(24) by taking into consideration that [23],

$\sum_{\omega=0}^{\infty}(-1)^{\omega} c_{\omega}^{(\alpha)}=(1-z)^{\alpha}$

So,

$S^{x}=(\Delta t)^{\alpha}\left(\frac{30}{12 h^{2}}-\frac{\alpha}{(\Delta t)^{\alpha}}-2^{\alpha}+1+\alpha(\Delta t)^{-\alpha}\right)-1$.

Since

$S \leq S\left(30+4 \sin ^{2}(\beta h)-32 \sin ^{2}\left(\frac{\beta h}{2}\right)\right)$,

and since

$S\left(30+4 \sin ^{2}(\beta h)-32 \sin ^{2}\left(\frac{\beta h}{2}\right)\right) \leq S^{x}$,

we use the sufficient condition for the present method to be stable is $S \leq S^{x}$ [22]. Therefore one is inside the stable region and gets reasonable numerical solution.

To verify the numerical accuracy in the event of initial value problems on the time interval $[0, T]$ we calculate the $E_{\max }$ at the time $t=T^{\prime}$, [20],

$$
E_{\max }=\max _{1 \leq i \leq N}\left|u_{\text {exact }}\left(x_{i}, T^{\prime}\right)-u_{\text {analytical }}\left(x_{i}, T^{\prime}\right)\right| \text {, }
$$

and the corresponding RMS error,

$$
E_{R M S}=\sqrt{\frac{1}{N} \sum_{i=1}^{N}\left(u_{\text {exact }}\left(x_{i}, T^{\prime}\right)-u_{\text {analytical }}\left(x_{i}, T^{\prime}\right)\right)^{2}} .
$$

In this work $N=10$, take $t=T^{\prime}=0.5$ and by the end of calculations we have,
$E_{\max }=0.0020$ and $E_{R M S}=0.0024$

\subsection{Numerical example}

Let us consider the fractional subdiffusion equation (1) with the boundary conditions

$U(0, t)=0, t \in[0,1]$,

$U(1, t)=0, t \in[0,1]$,

and the following initial conditions

$U(x, 0)=2 \sin (\pi x), x \in[0,1]$

The exact solution of this problem is given by [8],

$U(x, t)=\left(t^{2+\alpha}+t+2\right) \sin (\pi x)$.

For computational work, we have taken $h=\Delta x=0.1$

, $k=\Delta t=0.001, \alpha=0.9$ and choosen the suitable right-hand side function $f(x, t)$ such as,

$f(x, t)=\frac{1}{200} \frac{\sin (\pi x) \Gamma\left(\frac{9}{10}\right)\left(4959 t^{2} \pi+2.10 e 4 t^{(1 / 10)} \sin \left(\frac{\pi}{10}\right)\right)}{\pi}(32)$

$+\pi^{2} \sin (\pi x)\left(t^{\left(\frac{29}{10}\right)}+t+2\right)$.

Different appropriate functions $f(x, t)$ for each different alpha value are found by using the Maple algebraic system.

Table 1. Numerical results for $\alpha=0.85$.

\begin{tabular}{llll}
\hline \multicolumn{2}{l}{$\alpha=0.85$} & & \\
\hline $\mathrm{x}$ & $\begin{array}{l}\text { Exact } \\
\text { Solution }\end{array}$ & $\begin{array}{l}\text { Numerical } \\
\text { Solution }\end{array}$ & Error \\
\hline 0.0 & 0 & 0 & 0 \\
0.1 & 0.8154 & 0.8165 & 0.0011 \\
0.2 & 1.5510 & 1.5523 & 0.0013 \\
0.3 & 2.1348 & 2.1367 & 0.0019 \\
0.4 & 2.5095 & 2.5118 & 0.0023 \\
0.5 & 2.6387 & 2.6408 & 0.0021 \\
0.6 & 2.5095 & 2.5118 & 0.0023 \\
0.7 & 2.1348 & 2.1366 & 0.0018 \\
0.8 & 1.5509 & 1.5519 & 0.0010 \\
0.9 & 0.8155 & 0.8163 & 0.0008 \\
1.0 & 0 & 0 & 0 \\
\hline
\end{tabular}

In Table 1, we show exact and numerical solutions of fractional subdiffusion Eq.1 and we compare exact and numerical solution using absolute error at $t=0.5$ for $\alpha=0.85, \Delta x=0.1, \Delta t=0.001$. 
Table 2. Numerical results for $\alpha=0.90$.

\begin{tabular}{llll}
\hline \multicolumn{2}{l}{$\alpha=0.90$} & & \\
\hline $\mathrm{x}$ & $\begin{array}{l}\text { Exact } \\
\text { Solution }\end{array}$ & $\begin{array}{l}\text { Numerical } \\
\text { Solution }\end{array}$ & Error \\
\hline 0.0 & 0 & 0 & 0 \\
0.1 & 0.8139 & 0.8149 & 0.0010 \\
0.2 & 1.5482 & 1.5475 & 0.0007 \\
0.3 & 2.1310 & 2.1316 & 0.0006 \\
0.4 & 2.5051 & 2.5043 & 0.0008 \\
0.5 & 2.6340 & 2.6360 & 0.0020 \\
0.6 & 2.5050 & 2.5040 & 0.0010 \\
0.7 & 2.1310 & 2.1301 & 0.0009 \\
0.8 & 1.5482 & 1.5477 & 0.0005 \\
0.9 & 0.8140 & 0.8138 & 0.0002 \\
1.0 & 0 & 0 & 0 \\
\hline
\end{tabular}

In Table 2 we observe exact and numerical solutions of fractional subdiffusion Eq.1 and absolute error at $t=0.5$ for $\alpha=0.90, \Delta x=0.1, \Delta t=0.001$.

Table 3. Numerical results for $\alpha=0.95$.

\begin{tabular}{llll}
\hline \multicolumn{2}{l}{$\alpha=0.95$} & & \\
\hline $\mathrm{x}$ & $\begin{array}{l}\text { Exact } \\
\text { Solution }\end{array}$ & $\begin{array}{l}\text { Numerical } \\
\text { Solution }\end{array}$ & Error \\
\hline 0.0 & 0 & 0 & 0 \\
0.1 & 0.8125 & 0.8136 & 0.0011 \\
0.2 & 1.5455 & 1.5468 & 0.0013 \\
0.3 & 2.1272 & 2.1293 & 0.0021 \\
0.4 & 2.5007 & 2.5024 & 0.0017 \\
0.5 & 2.6294 & 2.6317 & 0.0023 \\
0.6 & 2.5007 & 2.5021 & 0.0014 \\
0.7 & 2.1273 & 2.1291 & 0.0018 \\
0.8 & 1.5455 & 1.5464 & 0.0009 \\
0.9 & 0.8126 & 0.8134 & 0.0008 \\
1.0 & 0 & 0 & 0 \\
\hline
\end{tabular}

In Table 3 we can see exact and numerical solutions of fractional subdiffusion Eq. 1 and absolute error at $t=0.5$ for $\alpha=0.95, \Delta x=0.1, \Delta t=0.001$.

Table 4. Numerical results for $\alpha=0.90$ at different times.

\begin{tabular}{lllllll}
\hline \multicolumn{3}{c}{$t=0.1$} & \multicolumn{5}{c}{$t=0.2$} \\
\hline $\mathrm{x}$ & $\begin{array}{l}\text { Exact } \\
\text { Solution }\end{array}$ & $\begin{array}{l}\text { Numerical } \\
\text { Solution }\end{array}$ & Error & $\begin{array}{l}\text { Exact } \\
\text { Solution }\end{array}$ & $\begin{array}{l}\text { Numerical } \\
\text { Solution }\end{array}$ & Error \\
\hline 0.0 & 0 & 0 & 0 & 0 & 0 & 0 \\
0.1 & 0.6827 & 0.6839 & 0.0012 & 0.6493 & 0.6510 & 0.0017 \\
0.2 & 1.2987 & 1.3008 & 0.0021 & 1.2351 & 1.2378 & 0.0027 \\
0.3 & 1.7874 & 1.7898 & 0.0024 & 1.7000 & 1.7037 & 0.0037 \\
0.4 & 2.1012 & 2.1043 & 0.0031 & 1.9984 & 2.0026 & 0.0042 \\
0.5 & 2.2094 & 2.2120 & 0.0026 & 2.1013 & 2.1054 & 0.0041 \\
0.6 & 2.1012 & 2.1041 & 0.0029 & 1.9984 & 2.0029 & 0.0045 \\
0.7 & 1.7875 & 1.7899 & 0.0024 & 1.7000 & 1.7034 & 0.0034 \\
0.8 & 1.2986 & 1.3009 & 0.0023 & 1.2351 & 1.2377 & 0.0026 \\
0.9 & 0.6828 & 0.6840 & 0.0012 & 0.6494 & 0.6510 & 0.0016 \\
1.0 & 0 & 0 & 0 & 0 & 0 & 0 \\
\hline
\end{tabular}

In the above table exact and numerical solutions of fractional subdiffusion Eq.1 and absolute error for different times for $\alpha=0.90, \Delta x=0.1, \Delta t=0.001$.

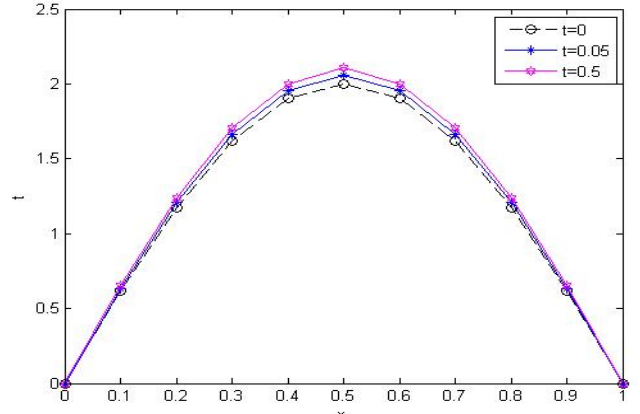

Figure 1. Numerical solution of Eq.(1) for various values of $t$.

In Figure1 we observe numerical solutions of fractional diffusion equation (Eq.1) at different times $t=0, t=0.05, t=0.5$ for $\alpha=0.90$, $\Delta x=0.1, \Delta t=0.001$.

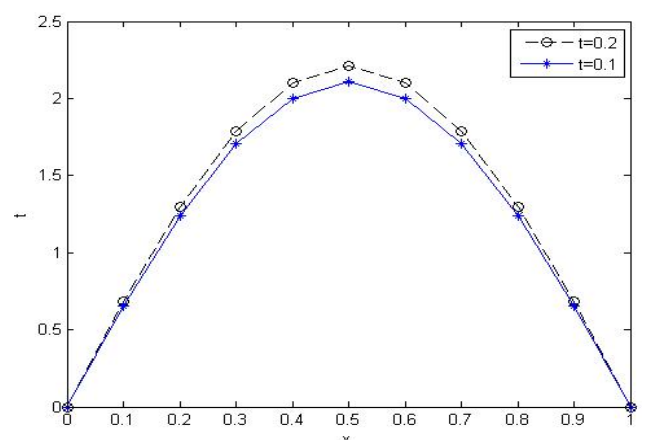

Figure 2. Numerical solution of Eq.(1) for various values of $t$.

In Fig. 2 we observe numerical solutions of fractional diffusion equation (Eq.1) at different times $t=0.1, t=0.2$ for $\alpha=0.90, \Delta x=0.1, \Delta t=0.001$. These solutions given by Table 4 .

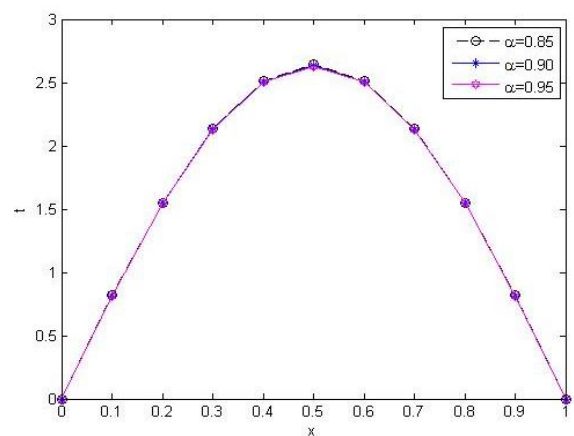

Figure 3. Numerical solution of Eq.(1) for various values of $\alpha$.

In Figure 3 we observe numerical solutions of fractional diffusion equation (Eq.(1)) for different orders $\alpha=0.85, \alpha=0.90$ and $\alpha=0.95$ at $t=0.5$ where $\Delta x=0.1, \Delta t=0.001$. The numerical solutions given by Table 1-3. 


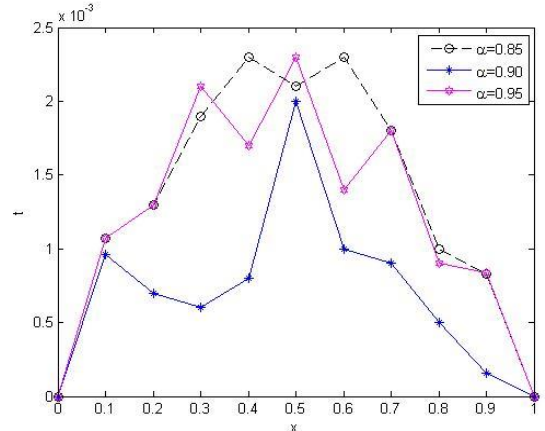

Figure 4. Absolute errors for various values of $\alpha$.

In Figure 4 we observe absolute errors of the numerical method for the Eq.(1) at $t=0.5$, for different orders $\alpha=0.85, \alpha=0.90$ and $\alpha=0.95$ where $\Delta x=0.1, \Delta t=0.001$.

\section{Conclusion}

We introduced numerical schemes for solving time fractional diffusion equation a new finite difference method which is an explicit method. This method involves fractional Riemann-Liouville derivatives, which were used with Grünwald Letnikov formula. In this study, we have used 5-point and 3-point difference scheme while discretizing of equation. For the all numerical algorithm we used Maple 15 algebraic system with acceptable CPU time and for the graphics of the solution Matlab 2009 has been used. On the other hand it was also used that a fractional von-Neumann stability analysis which leads to correct estimate of the stability conditions for diffusion equations. According to this analysis we found a stability bound on $S=(\Delta t)^{\alpha} / 12 h^{2}$. Furthermore we investigated absolute and RMS errors, they are compatible. The numerical and exact solutions of Eq.(1) at several times and for different orders have been shown in tables and solutions were supported by the graphics. These results show that the present method gives us new solutions in agreement with the analytical solutions. Hence, we conclude that the proposed approach is very powerful and efficient to investigate fractional differential equation numerically.

\section{Acknowledgments}

The authors would like to thank the anonymous reviewers for their valuable comments.

\section{References}

[1] Ciesielski, M., Leszczynski J. (2003). Numerical simulations of anomalous diffusion, Computer Methods in Mechanics, Gliwice, Poland, June 3-6.

[2] Luenberger, D.G., (1973). Introduction to Linear and Nonlinear Programming. Addison-Wesley, California

[3] Berkowitz, B., Scher, H., (1997). Anomalous transport in random fracture networks, Physical Review Letters, 79, 4038-4041.

[4] Klemm, A., Müller, H.P., Kimmich R., (1997). NMR-microscopy of pore-space backbones in rock, Phys. Rev. E, 55,4413.

[5] Crank, J., (1989). The Mathematics of Diffusion 2nd ed., Oxford University Press.

[6] Palade, L.I., Attane, P., Huilgol, R.R., Mena, B., (1999). Anomalous stability behavior of a properly invariant constitutive equation which generalises fractional derivative models, International Journal of Engineering Science., 37, 315-329.

[7] Amblard, F., Maggs, A.C., Yurke, B.,.Pargellis, A.N., Leibler, S., (1996). Subdiffusion and anomalous local viscoelasticity in actin networks, Physical Review Letters, 77, 4470-4473.

[8] Baskonus, H.M., Bulut, H., (2015). On the Numerical Solutions of Some Fractional Ordinary Differential Equations by Fractional AdamsBashforth-Moulton Method, Open Mathematics, 13(1), 547-556.

[9] Baskonus, H.M., Mekkaoui, T., Hammouch, Z. and Bulut, H., (2015). Active Control of a Chaotic Fractional Order Economic System, Entropy, 17(8), 5771-5783.

[10] Baskonus, H.M., Bulut, H., (2016).Regarding on the prototype solutions for the nonlinear fractionalorder biological population model, AIP Conference Proceedings 1738, 290004.

[11] Baskonus, H.M., Hammouch, Z., Mekkaoui, T. and Bulut, H., (2016).Chaos in the fractional order logistic delay system: Circuit realization and synchronization, AIP Conference Proceedings, 1738, 290005.

[12] Gencoglu, M. T., Baskonus, H. M. and Bulut, H.,(2017).Numerical simulations to the nonlinear model of interpersonal Relationships with time fractional derivative, AIP Conference Proceedings, 1798, 1-9 (020103).

[13] Li C., Zeng F., (2015). Numerical Methods for Fractional Calculus, Taylor \& Francis Group.

[14] Nakagawa, J., Sakamoto K., Yamamoto M., (2010). Overwiev to mathematical analysis for fractional diffusion equation-new mathematical aspects motivated by industrial collaboration, Journal of Math-for-Industry, 2, 99-108.

[15] Narahari Achar B., Hanneken J., (2004). Fractional radial diffusion in a cylinder, Journal of Molecular Liquids, 114, 147-151.

[16] Langlands, T., (2006). Fractional Dynamics, Physica A 367, 136

[17] Diethelm, K., Ford, J., Freed, A., Luchko, Y., (2005). Algorithms for the fractional calculus: a selection of numerical methods, Computer Methods in Applied Mechanics and Engineering, 194(6), 743-773. 
[18] He, J.H., (1998). Approximate analytical solution for seepage flow with fractional derivatives in porous media, Computer Methods in Applied Mechanics and Engineering, 167, 57-68.

[19] Kurulay, M., Bayram, M., (2009). Approximate analytical solution for the fractional modified $\mathrm{KdV}$ by differential transform method, Communications in Nonlinear Science and Numerical Simulation, 15(7), 1777-178.

[20] Reutskiy, S. Yu., A. (2017). New semi-analytical collocation method for solving multi-term fractional partial differential equations with time variable coefficients, Applied Mathematical Modelling, 45, 238-254.

[21] Smith, G. D., (1985). Numerical Solution of Partial Difference Equations, Finite Difference Methods, Oxford University.

[22] Yuste, S. B., Acedo, L., (2005). An explicit finite difference method and a new Von Neumann-type stability analysis for fractional diffusion equations, Society for Industrial and Applied Mathematics, 42(5), 1862-1874.
[23] Murillo, J. Q., Yuste, S. B., (2011). An Explicit Numerical Method for the Fractional Cable Equation, Hindawi Publishing Corporation International Journal of Differential Equations.

[24] Lubich, C.,(1986).Discretized fractional calculus, SIAM Journal on Mathematical Analysis, 17(3),704-719.

Dilara Altan Koç is currently a Research Assistant and Ph.D. student in the Department of Mathematics in Mugla Sitki Kocman University, Turkey. She obtained her B.Sc. and M.Sc. degrees from Mugla Sitki Kocman University. Her research interests include numerical methods for partial differential equations with fractional order.

Mustafa Gülsu is a full Professor at the Department of Mathematics, Mugla Sitki Kocman University, Turkey. He received his B.Sc. from University of Marmara, M.Sc. from Northeastern University and PhD. from Ege University. His research areas include fractional differential equation and fractional calculus.

An International Journal of Optimization and Control: Theories \& Applications (http://ijocta.balikesir.edu.tr)

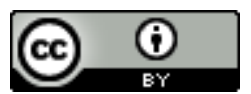

This work is licensed under a Creative Commons Attribution 4.0 International License. The authors retain ownership of the copyright for their article, but they allow anyone to download, reuse, reprint, modify, distribute, and/or copy articles in IJOCTA, so long as the original authors and source are credited. To see the complete license contents, please visit http://creativecommons.org/licenses/by/4.0/. 